\title{
The association of short sleep after acute coronary syndrome with recurrent cardiac events and mortality
}

\author{
Carmela Alcántara, Ph.D., James Peacock, M.D., Karina W. Davidson, Ph.D., David Hiti, \\ M.A., and Donald Edmondson, Ph.D., M.P.H. \\ Columbia University Medical Center, New York, NY.
}

\section{Keywords}

sleep; myocardial infarction; death; acute coronary syndrome

\begin{abstract}
Although most patients survive an acute coronary syndrome [ACS myocardial infarction (MI) or unstable angina (UA)], within 5 years $22 \%$ will be re-hospitalized and more than 1 in 3 will die. ${ }^{1}$ Thus, identifying modifiable risk factors for ACS recurrence and mortality is critical.
\end{abstract}

\begin{abstract}
Short sleep duration (less than 7 hours per night), is associated with a range of physiologic responses (eg, inflammation, impaired glucose tolerance, reduced insulin sensitivity, and appetite regulation), health behaviors, and comorbidities (eg, poor diet, diabetes, metabolic syndrome, obesity, and hypertension) that increase risk of coronary heart disease (CHD)..$^{2-6}$ A meta-analysis of prospective cohort studies found that self-reported short sleep was associated with a $48 \%$ increased risk of incident CHD. ${ }^{5}$ Short sleep is also common among post-ACS patients. ${ }^{7,8}$ However, no study has examined whether short sleep is associated with risk in ACS patients. We hypothesized that ACS patients who report short sleep duration in the month after ACS would be at increased risk for 1-year ACS recurrence and mortality relative to those who report sleeping 7 hours or more per night.
\end{abstract}

\section{Methods}

Consecutively hospitalized patients with ACS were enrolled between February 1, 2009, and March 31, 2012, in the single-site, prospective, observational cohort Prescription Use, Lifestyle and Stress Evaluation (PULSE) study ( $\mathrm{N}=866$ ). Participants completed the Pittsburgh Sleep Quality Index (PSQI), ${ }^{9}$ a widely used self-report measure of sleep during the past month. Responses to the item, "During the past month, how many hours of actual sleep did you get at night?," were dichotomized as less than 7 hours (short sleep) or 7 hours or more..$^{5}$ The primary composite endpoint was first ACS recurrence (hospitalization for

\footnotetext{
(C) 2013 Elsevier Ireland Ltd. All rights reserved.

Correspondence concerning this article should be addressed to Carmela Alcántara Ph.D., Center for Behavioral Cardiovascular Health, Department of Medicine, Columbia University Medical Center; PH-9, Room 9-319; 622 West $168^{\text {th }}$ Street; New York, NY 10032 , U.S.A.ca2543@cumc.columbia.edu; Phone: 212-342-5503; Fax: 212-342-3431..

Publisher's Disclaimer: This is a PDF file of an unedited manuscript that has been accepted for publication. As a service to our customers we are providing this early version of the manuscript. The manuscript will undergo copyediting, typesetting, and review of the resulting proof before it is published in its final citable form. Please note that during the production process errors may be discovered which could affect the content, and all legal disclaimers that apply to the journal pertain.

The authors take responsibility for all aspects of the reliability and freedom from bias of the data presented and their discussed interpretation.

There are no conflicts of interest to report.
} 
nonfatal MI or unstable angina) or death within 1-year of their index ACS hospitalization. Patient-reported re-hospitalizations were adjudicated from hospital records by boardcertified cardiologists. The Social Security National Death Index was searched to verify vital status.

Cox proportional hazards regression models were used to determine the association of short sleep with 1-year ACS recurrence or mortality. Model 1 estimated the unadjusted association between short sleep and the composite outcome. Model 2 included additional adjustment for self-reported age, sex, race, years of education, left ventricular ejection fraction (LVEF) less than 40\% (abstracted from the medical record), Charlson comorbidity index, and Global Registry of Acute Coronary Events (GRACE) risk score. Model 3 further adjusted for baseline depressive symptoms, body mass index (BMI), past-month sleep medications use, past-month breathing-related sleep disturbance, and perceived sleep quality (measured with the PSQI).

Participants who experienced ACS recurrence or death before 1 month $(n=8)$ and those with incomplete data $(\mathrm{n}=116)$ were excluded. Parameter estimates using multiply imputed data did not differ appreciably from complete data estimates. Complete data were available for 742 patients.

\section{Results}

Participants who reported short sleep were significantly younger, were more likely to be women, had lower GRACE risk scores, reported higher depressive symptoms, and were more likely to report past-month use of sleep medications and past-month breathing-related sleep disturbances than their counterparts with longer sleep (Table 1). Overall, 127 participants had a primary end point (31 MI cases, 78 UA cases, and 18 deaths) during 1year follow-up. In this sample, 76 participants $(20.1 \%)$ who reported short sleep had a primary end point compared with $51(14.0 \%)$ who reported at least 7 hours of sleep.

Short sleep was associated with an increased 1-year risk of ACS recurrence or mortality in the unadjusted model (hazard ratio [HR] $=1.52 ; 95 \% \mathrm{CI}, 1.06-2.17$ ) and remained significant after adjustment for demographics and clinical disease severity (HR=1.50; 95\% CI, 1.05-2.16) (Figure). Black race (HR=1.96; 95\% CI, 1.32-2.89) and Charlson comorbidity index (HR=1.22; 95\% CI, 1.11-1.34) were the only significant covariates in this model. Further adjustment for baseline depressive symptoms, BMI, past-month sleep medication use, and past-month breathing-related sleep disturbances did not alter the association of short sleep with poor prognosis ( $\mathrm{HR}=1.52 ; 95 \% \mathrm{CI}, 1.06-2.18)$. A sensitivity analysis with further adjustment for perceived subjective sleep quality, measured with the global PSQI score, did not alter the association ( $\mathrm{HR}=1.60 ; 95 \% \mathrm{CI}, 1.05-2.44)$.

\section{Conclusion}

Our results suggest that more than half of ACS patients may experience short sleep duration (sleeping $<7$ hours per night) in the month after ACS, and that short sleep is associated with a more than 50\% increased risk of ACS recurrence or death in the year after their index ACS event. The association of short sleep with recurrent ACS and mortality was consistent and independent of demographics, disease severity, comorbidities, depressive symptoms, BMI, sleep medication use, and breathing-related sleep disturbances.

Although short sleep has been linked to risk factors for CHD (eg, endothelial dysfunction, elevated C-reactive protein level, and metabolic dysregulation), ${ }^{5,7}$ the underlying pathophysiologic mechanisms and the direction of causality are not yet known. Future research should identify the biological and psychosocial mechanisms by which short sleep 
confers excess risk of poor prognosis and whether short sleep can be modified to improve prognosis. For example, short sleep might deplete an individual's self-regulation resources, which might in turn inhibit an individual's capacity to perform health-promoting behaviors, which might place him or her at increased risk for ACS recurrence and mortality. ${ }^{10}$

Our study is not without its limitations. First, we used a self-report measure of sleep duration. However, unless participants differentially misclassified their sleep duration based on their risk for recurrence and mortality, error responses would have biased our results toward no effect for short sleep. Relatedly, we used self-reported breathing-related sleep disturbances as a proxy for sleep apnea; thus, we were only able to adjust for confounding due to the presence of sleep-disordered breathing that was known to participants. Second, we could determine average sleep duration only for the month after the index hospitalization. In sum, short sleep in the month after ACS appears to confer risk of ACS recurrence and mortality, independent of likely confounders. Future research should use prospective designs with objective measures of sleep, and test whether short sleep is modifiable in ACS patients and whether interventions can offset the risk associated with it.

\section{Acknowledgments}

This research was supported by the National Institutes of Health grants HL088117 and HL084034 awarded to Dr Davidson, HL115941-01S1 awarded to Dr Alcántara, HL47540 awarded to Dr Edmondson, and T32HL007854-16 awarded to Dr Peacock. The contents of this manuscript are solely the responsibility of the authors and do not necessarily represent the official view of the National Institutes of Health.

\section{References}

1. Roger VL, Go AS, Lloyd-Jones DM, et al. Heart disease and stroke statistics--2012 update: a report from the American Heart Association. Circulation. 2012; 125:e2-20. [PubMed: 22179539]

2. Luyster FS, Strollo PJ Jr. Zee PC, Walsh JK. Sleep: a health imperative. Sleep. 2012; 35:727-34. [PubMed: 22654183]

3. Knutson KL, Van Cauter E, Rathouz PJ, et al. Association between sleep and blood pressure in midlife: the CARDIA sleep study. Arch Intern Med. 2009; 169:1055-61. [PubMed: 19506175]

4. King CR, Knutson KL, Rathouz PJ, Sidney S, Liu K, Lauderdale DS. Short sleep duration and incident coronary artery calcification. JAMA. 2008; 300:2859-66. [PubMed: 19109114]

5. Cappuccio FP, Cooper D, D'Elia L, Strazzullo P, Miller MA. Sleep duration predicts cardiovascular outcomes: a systematic review and meta-analysis of prospective studies. Eur Heart J. 2011; 32:1484-92. [PubMed: 21300732]

6. Broussard JL, Ehrmann DA, Van Cauter E, Tasali E, Brady MJ. Impaired insulin signaling in human adipocytes after experimental sleep restriction: a randomized, crossover study. Ann Intern Med. 2012; 157:549-57. [PubMed: 23070488]

7. Wolk R, Gami AS, Garcia-Touchard A, Somers VK. Sleep and cardiovascular disease. Curr Probl Cardiol. 2005; 30:625-62. [PubMed: 16301095]

8. Shaffer J, Kronish I, Burg M, Clemow L, Edmondson D. Association of Acute Coronary SyndromeInduced Posttraumatic Stress Disorder Symptoms with Self-Reported Sleep. Ann Behav Med. May 30.2013 [Epub ahead of print].

9. Buysse DJ, Reynolds CF 3rd, Monk TH, Berman SR, Kupfer DJ. The Pittsburgh Sleep Quality Index: a new instrument for psychiatric practice and research. Psychiatry Res. 1989; 28:193-213. [PubMed: 2748771]

10. Hagger MS, Wood C, Stiff C, Chatzisarantis NLD. The strength model of self-regulation failure and health-related behaviour. Health Psychol Rev. 2009; 3:208-38. 


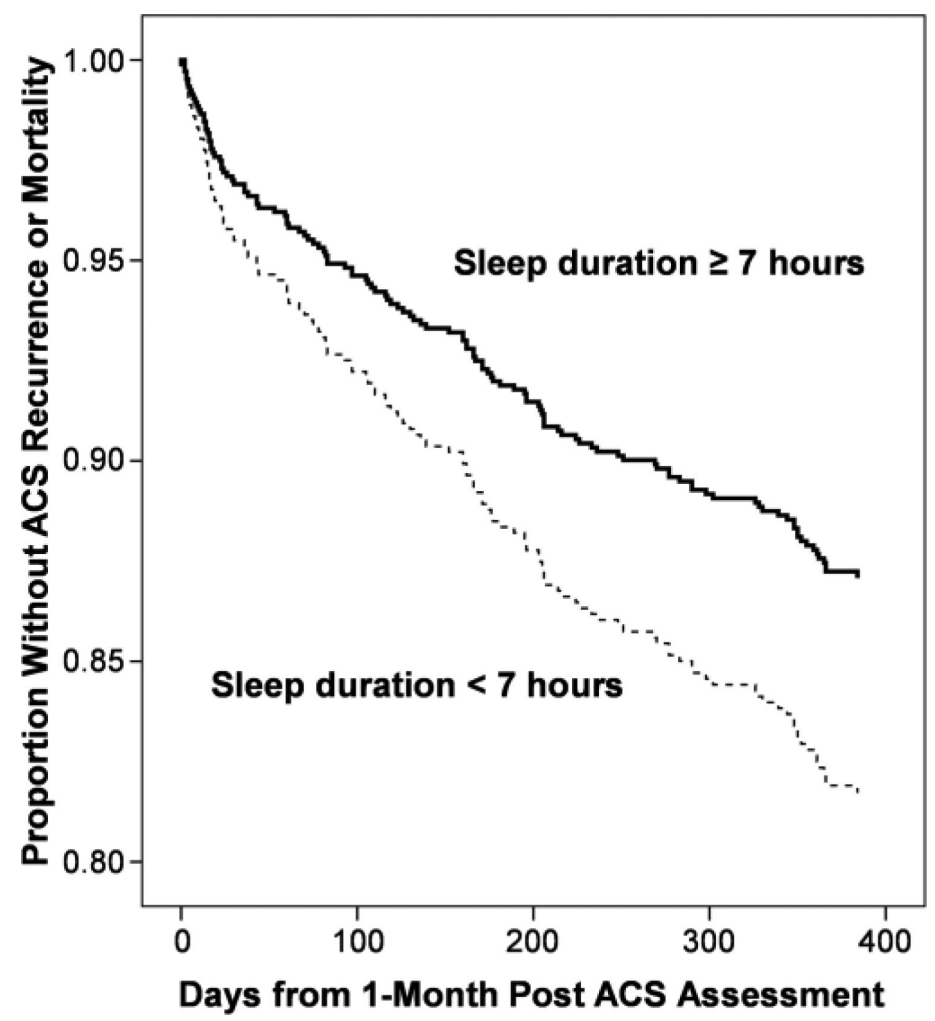

Figure 1.

Cox proportional hazards regression analysis predicted curve for acute coronary syndrome (ACS) patients by sleep duration, adjusted for age, sex, race, years of education, Charlson comorbidity index, Global Registry of Acute Coronary Events risk score, and left ventricular ejection fraction. 
Table 1

Sociodemographic and Medical Characteristics of Post-ACS Patients by Sleep Duration, PULSE $(\mathrm{N}=742)^{a}$

\begin{tabular}{|c|c|c|c|}
\hline Characteristic & Sleep $\geq 7$ hours $(n=364)$ & Sleep $<7$ hours $(n=378)$ & $P$ \\
\hline \multicolumn{4}{|l|}{ Demographics } \\
\hline Age, y & $64.62(11.03)$ & $62.34(11.60)$ & .006 \\
\hline Female, No. (\%) & $110(30.2)$ & $142(37.6)$ & .04 \\
\hline Educational level, y & $13.24(4.36)$ & $13.20(4.17)$ & .91 \\
\hline Black, No. $(\%)$ & $73(20.1)$ & $91(24.1)$ & .19 \\
\hline \multicolumn{4}{|l|}{ Clinical Severity } \\
\hline Charlson comorbidity index & $1.70(1.69)$ & $1.58(1.65)$ & .35 \\
\hline GRACE & 93.78 (29.09) & $89.39(30.17)$ & .04 \\
\hline LVEF < $40 \%$, No. $(\%)$ & $49(13.5)$ & $47(12.4)$ & 68 \\
\hline \multicolumn{4}{|l|}{ Behavioral, Psychosocial, and Sleep Factors } \\
\hline Body mass index & $28.92(5.56)$ & $29.61(5.86)$ & .10 \\
\hline Depressive symptoms & $7.33(6.78)$ & $9.65(7.11)$ & $<.001$ \\
\hline Past-month sleep medication use, No. (\%) & $53(14.6)$ & $90(23.9)$ & .001 \\
\hline Past-month breathing-related sleep disturbance, No. (\%) & $46(12.7)$ & $91(24.2)$ & $<.001$ \\
\hline \multicolumn{4}{|l|}{ Outcome } \\
\hline ACS recurrence or death, No. $(\%)$ & $51(14.0)$ & $76(20.1)$ & .03 \\
\hline Days to ACS recurrence or death & $351.11(96.89)$ & $339.08(107.77)$ & .11 \\
\hline
\end{tabular}

Abbreviations: ACS, acute coronary syndrome; GRACE, Global Registry of Coronary Events; LVEF, left ventricular ejection fraction; PULSE, Prescription Use, Lifestyle, and Stress Evaluation study.

${ }^{a}$ Data are presented as mean (SD) unless otherwise indicated. Sleep duration was assessed at baseline. ACS recurrence includes hospitalization for nonfatal MI or unstable angina. 